\title{
Computer simulations for direct conversion of the HF electromagnetic wave into the upper hybrid wave in ionospheric heating experiments
}

\author{
H. O. Ueda ${ }^{1}$, Y. Omura ${ }^{2}$ and H. Matsumoto ${ }^{2}$ \\ ${ }^{1}$ Department of Urban Environment and Systems, Chiba University 1-33 Yayoi, Inage, Chiba 263, Japan \\ ${ }^{2}$ Radio Atmospheric Science Center, Kyoto University Uji, Kyoto 611, Japan
}

Received: 6 October 1997/ Revised: 30 April 1998/Accepted: 13 May 1998

\begin{abstract}
Excitation of upper hybrid waves associated with the ionospheric heating experiments is assumed to be essential in explaining some of the features of stimulated electromagnetic emissions (SEE). A direct conversion process is proposed as an excitation mechanism of the upper hybrid waves where the energy of an obliquely propagating electromagnetic pump wave is converted into the electrostatic upper hybrid waves due to small-scale density irregularities. We performed electromagnetic particle-in-cell simulations to investigate the energy conversion process in the ionospheric heating experiments. We studied dependence of the amplitude of the excited wave on the propagation angle of the pump wave, scale length of the density irregularity, degree of the irregularity, and thermal velocity of the plasma. The maximum amplitude is found to be $37 \%$ of the pump amplitude under an optimum condition.
\end{abstract}

Key words. Ionosphere (ionospheric irregularities; plasma waves and instabilities; wave-particle interactions).

\section{Introduction}

Investigations of the interaction of a high-frequency powerful radio wave of $\mathrm{O}$ mode (L-O mode) polarization with the ionospheric F- region plasma show that secondary stimulated electromagnetic emissions (SEE) are generated in the heated ionospheric region (Thide et al., 1989; Leyser et al., 1989, 1990, 1993, 1994). They are observed in the altitude range between the reflection height of a pump wave and the level of the upper hybrid

Correspondence to: H. O. Ueda
(UH) resonance, which is located several kilometers below the reflected height. The SEE result from reemission of HF-induced waves as electromagnetic waves due to some wave-wave interactions. The excitation of $\mathrm{UH}$ waves associated with the heating was thought to be essential in explaining some of the features of SEE (Leyser et al., 1989, 1993; Goodman et al., 1993). The SEE are observed only during L-O mode pumping in narrow ranges of the pump frequency, near several harmonics of the electron cyclotron frequency. The SEE grows on the same time scale as that for a thermally generated field-aligned density irregularity to cause the HF radar backscattering (Hedberg et al., 1983; Kagan and Frolov, 1996)

The electrostatic waves at the $\mathrm{UH}$ resonance were assumed to be excited via "direct conversion" (DC) of the energy of an obliquely propagating electromagnetic pump wave into that of the electrostatic wave through a small-scale density irregularity whose scale length perpendicular to the geomagnetic field is comparable to or much less than the wave length of the pump wave (Wong et al., 1981; Morales et al., 1985). It was shown that the DC is a fundamental property of wave processes in a nonuniform plasma, and that it occurs whenever appropriate frequency and wave number matching conditions are satisfied. The DC is basically a linear process and has no threshold, so that is different from a usual parametric process, and is distinct from the conventional linear mode conversion process that occurs independent of any plasma resonance such as cyclotron harmonics. The possibility of UH wave excitation by the DC in the ionospheric heating experiments was discussed theoretically by Antani et al. (1991, 1995). It is derived by the linear analysis that the amplitude of the excited UH wave is proportional to $|D|^{-1}$, where $D$ is the dielectric function of the UH wave. This indicates that a large amplification can occur if $D$ is close to zero. The conditions must be subject to the wavelength which almost equals to the scale length of the irregularity, the propagation angle to the geomagnetic field, etc. However it is difficult to evaluate the amplitude quantita- 
tively by this theory because of the complexity of the oblique propagation in the inhomogeneous plasma and possible nonlinear effects.

The amplitude of the excited UH wave should be an important factor for subsequent generation mechanisms of the SEE. Electromagnetic particle-in-cell simulations are quite useful when studying the DC process, but it was never approached by this simulation. Therefore we carried out simulations using the model of the ionospheric heating experiments. We reproduce the physical property of the energy conversion process in the simulation to clarify the dependence of the amplitude of the excited $\mathrm{UH}$ waves on various ionospheric and heating parameters.

The work is organized as follows. In the second section the physical model of the DC process is described. In the third section, we show the computer simulation model and discussions based on the results of the simulations. Finally, in the fourth section, summary and concluding remarks are given.

\section{Theoretical model}

An essential physical process of the DC is a coupling between the high-frequency oscillatory velocity imposed on the plasma particles by the pump wave and the lowfrequency density fluctuation, which generates a beat current that plays the role of a radiating antenna (Morales et al., 1985). For the ionospheric heating experiment, the following theoretical model has been given by Antani et al. (1991).

Figure 1 shows a schematic illustration of a typical ionospheric heating experiment. A high-power L-O mode electromagnetic pump wave, whose frequency and wave number are $\omega_{0}$ and $k_{0}$, respectively, is vertically launched into the ionospheric F-region. Here, we define the term ' $\mathrm{L}-\mathrm{O}$ mode wave' as a wave obliquely propagating to the external magnetic field, the polarization is left elliptical, and the frequency is higher than the plasma frequency. The pump wave is considered to be a high-frequency wave. The incident wave passes through

the $\mathrm{UH}$ resonance layer where $\omega_{0} \simeq \omega_{U H}=$ $\sqrt{\omega_{p e}^{2}+\Omega_{e}^{2}}, \omega_{U H}, \omega_{p e}$, and $\Omega_{e}$ are the UH frequency, the local plasma frequency, and the electron cyclotron frequency, respectively. Observations support the assumptions that a coherent quasi-neutral field-aligned density irregularity exists in the UH resonance layer. The density irregularity is defined by

$n_{1}=\tilde{n}_{1} \exp \left[i\left(\boldsymbol{k}_{\boldsymbol{n}} \cdot r-\omega_{n} t\right)\right]+$ c.c.

where $\omega_{n} \ll \omega_{o}, k_{n} \gg k_{o}$, and $\boldsymbol{k}_{n} \cdot \boldsymbol{B}_{e x}=0, \boldsymbol{B}_{e x}$ are the external geomagnetic field. The plasma electrons are initialized with velocities of sloshing motion supporting the pump wave.

$\boldsymbol{v}_{\boldsymbol{o}}=\frac{-\boldsymbol{e} \tilde{\boldsymbol{E}}_{\boldsymbol{o}}}{i \omega_{o} m} \exp \left[i\left(\boldsymbol{k}_{\boldsymbol{o}} \cdot r-\omega_{o} t\right)\right]+c . c$.

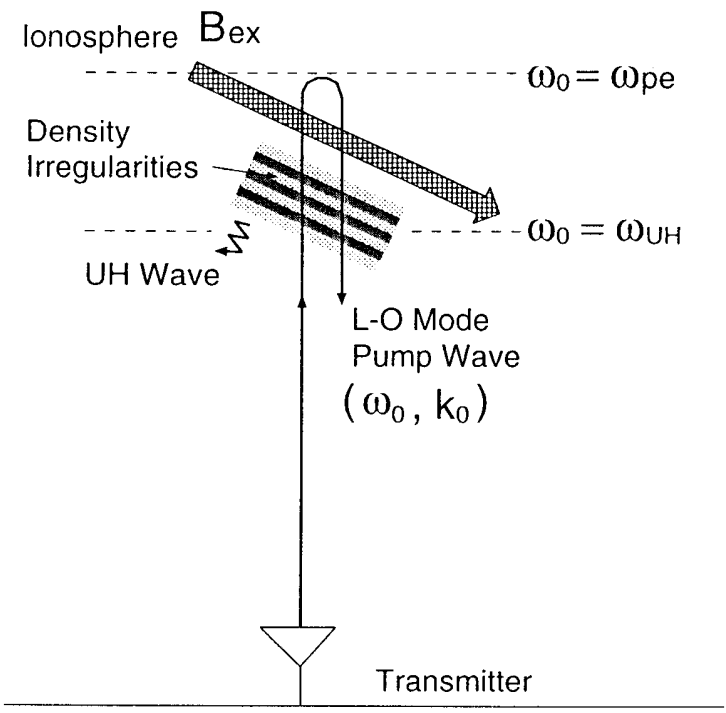

Fig. 1. Schematic illustration of the ionospheric heating experiment. The pump wave is a L-O mode wave, which is converted to an upper hybrid wave though the density irregularities along the magnetic field

where $-e$ and $m$ are the charge and mass of electrons, the constant vector amplitude $\tilde{\boldsymbol{E}}_{o}$ has perpendicular components to the geomagnetic field while a parallel component is the main factor of the electric field. Coupling between the oscillatory velocity and the density perturbation gives rise to a high-frequency source current density,

$\boldsymbol{J}_{\boldsymbol{s}}=-e n_{1} \boldsymbol{v}_{\mathrm{o}}$

that plays a role of an antenna. Resonant excitation occurs when the following frequency and wave number matching conditions are satisfied.

$\omega=\omega_{o} \pm \omega_{n}, \boldsymbol{k}=\boldsymbol{k}_{\boldsymbol{o}} \pm \boldsymbol{k}_{\boldsymbol{n}}$

where $\omega$ and $\boldsymbol{k}$ are frequency and wave number of the excited wave.

The self-consistent electric field $\boldsymbol{E}(\boldsymbol{k}, \omega)$ is determined from the driven Poisson equation,

$\nabla \cdot \boldsymbol{\epsilon} \cdot \boldsymbol{E}=\rho_{s}$

where $\epsilon$ is a kinetic plasma dielectric tensor, and $\rho_{s}$ is a source charge density associated with the current density as follows:

$\rho_{s}=\frac{1}{i \omega} \nabla \cdot \boldsymbol{J}_{\boldsymbol{s}}$.

Amplitude of the excited UH wave is obtained from the Eqs. (5) and (6)

$E(\boldsymbol{k}, \omega)=\frac{1}{i \omega} \frac{\boldsymbol{k} \hat{\cdot} \boldsymbol{J}_{s}}{D(\boldsymbol{k}, \omega)}$

where $D(\boldsymbol{k}, \omega)$ is a dielectric function for Bernstein modes because one of the branches must be the UH mode, and $\hat{\boldsymbol{k}}$ is a unit vector in the direction of $\boldsymbol{k}$ and also $\boldsymbol{E}$. The normalized amplitude is expressed as: 
$\frac{|E|}{\left|E_{o}\right|}=\left(\frac{\omega_{p e}^{2}}{\omega_{o} \omega}\right)\left(\frac{n_{1}}{n_{o}}\right) \frac{|\cos \theta|}{|D|}$

where $n_{o}$ is the average density of the plasma electrons, and $\theta$ is the propagation angle from the direction of the pump electric field that is close to the direction of the geomagnetic field.

It is important to note that a large amplitude of the excited electric fields can be reached if $|D|^{-1}$ is close to zero. The parameters $\lambda=k_{\perp}^{2} r_{e}^{2}$ and $\xi \equiv \omega /\left(\sqrt{2} k_{\|} r_{e}\right)$ change the absolute quantity of the dielectric function as is well known, where $k_{\perp}$ and $k_{\|}$are perpendicular and parallel components of $\boldsymbol{k}$ to the geomagnetic field, respectively, and $r_{e}$ is the electron Larmor radius defined as $v_{t e} /\left|\Omega_{e}\right|, v_{t e}$ is the perpendicular electron thermal velocity. Equation (7) shows that the energy conversion from the pump wave into the UH wave depends on the degree of the irregularity $\left(n_{1} / n_{o}\right)$, the propagation angle of the pump wave from the geomagnetic field that is related to $k_{\perp}$ and $k_{\|}$, the scale length of the irregularity as compared with the Larmor radius because $k_{\perp} \simeq k_{n} \gg k_{o \perp}$, and also the pump frequency.

We show results of numerical calculation of Eq. (7) in the next section, and compare with results of many simulation runs with various combinations of these parameters to study the characteristics of the excitation process of the UH wave.

\section{Computer simulations}

We first performed a 2-dimensional simulation to confirm the excitation of the UH wave for the case where the matching condition is satisfied. The simulation code is a typical $2 \frac{1}{2} \mathrm{D}$ PIC code-named KEMPO (Matsumoto and Omura, 1984). Simulation space in the code is 2-dimensional ( $x$ and $y$ ), while any quantity such as electric field, magnetic field, current density, and also particle velocity have 3-dimensional components. Figure

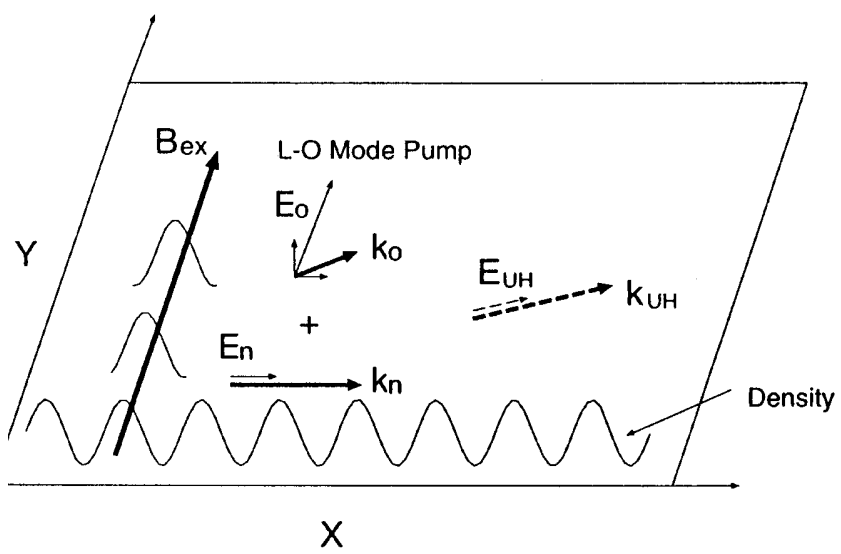

Fig. 2. Model of $2 \mathrm{D}$ electromagnetic particle simulation with periodic boundaries in $x$ - and $y$-axes. The external magnetic field is taken along the $y$-axis. A density irregularity with coherent variation in the perpendicular direction ( $x$-axis) and an oblique L-O mode wave are assumed as the initial condition

2 shows the model of the simulation. A coherent fieldaligned density irregularity with a characteristic wave number $k_{n}$ is pre-arranged uniformly in the simulation space. An obliquely propagating pump wave is set up as the initial condition. The wave number perpendicular to the geomagnetic field $k_{o \perp}$ is much larger than the parallel wavenumber $k_{o \|}$ and also $k_{o \perp} \ll k_{n}$. The frequency of the pump wave $\omega_{o}$ is assumed to correspond to the $\mathrm{UH}$ branch of the Bernstein modes so that the quasi-static density irregularity can satisfy the resonance condition. The pump frequency, wave number and polarization are determined to satisfy the linear dispersion relation for cold and uniform plasma. Three components $(x, y$, and $z$ components) of the electric and magnetic fields of the wave and the consistent electron velocity are given, respectively. The electron density distribution, however, does not include the effect of the pump wave. This gives rise to a backscattered wave with the wave number of $-\boldsymbol{k}_{\boldsymbol{o}}$ and the identical frequency $\omega_{o}$ at the start of the
Table 1. Parameters for simulations

\begin{tabular}{llll}
\hline Parameter & & $2 \mathrm{D}$ run & $1 \mathrm{D}$ runs \\
\hline Time step & $\Delta_{t}$ & $0.01 / \Omega_{e}$ & $0.005 / \Omega_{e}$ \\
Number of time step & $N_{\text {time }}$ & 2048 & 4096 \\
Electron plasma frequency & $\omega_{p e}$ & $4.0 \Omega_{e}$ & $4.0 \Omega_{e}$ \\
Ion plasma frequency & $\omega_{p i}$ & $0.2 \Omega_{e}$ & $0.2 \Omega_{e}$ \\
Upper hybrid frequency & $\omega_{U H}$ & $4.12 \Omega_{e}$ & $4.12 \Omega_{e}$ \\
Pump frequency & $\omega_{o}$ & $4.65 \Omega_{e}$ & $4.60 \Omega_{e}$ \\
Grid spacing & $\Delta_{x},\left(\Delta_{y}\right)$ & $1.67 \times 10^{-2} c / \Omega_{e}$ & $6.67 \times 10^{-3} c / \Omega_{e}$ \\
Number of grid points & $N_{x},\left(N_{y}\right)$ & 1024 & 8192 \\
Parallel pump wave length & $2 \pi / k_{o \|}$ & $1024 \Delta_{y}$ & \\
Perpendicular pump wave length & $2 \pi / k_{o}$ & $256 \Delta_{x}$ & \\
Pump wave length & $2 \pi / k_{o}$ & & $1024 \Delta_{x}$ \\
Propagation angle of pump wave & & $76^{\circ}$ & $70^{\circ} \sim 85^{\circ}$ \\
Scale length of irregularity & $2 \pi / k_{n}$ & $32 \Delta_{x}$ & $32 \Delta_{x} \sim 256 \Delta_{x}$ \\
Electron thermal velocity & $v_{t e}$ & $6.6 \times 10^{-2} c$ & $5.3,2.0 \times 10^{-2} c$ \\
Ion thermal velocity & $v_{t i}$ & $3.3 \times 10^{-3} c$ & $2.6,1.0 \times 10^{-3} c$ \\
Mass ratio of ion to electron & $m_{i} / m_{e}$ & 400 & 400 \\
Number of super particles for electron & $N_{p e}$ & $8 \times N_{x} \times N_{y}$ & $16 \times N_{x}$ \\
Number of super particles for ion & $N_{p i}$ & $8 \times N_{x} \times N_{y}$ & $16 \times N_{x}$ \\
Degree of irregularity & $\tilde{n}_{1}$ & $0.2 n_{o}$ & $0.05 \sim 0.25 n_{o}$ \\
Amplitude of pump magnetic field & $B_{o}$ & $0.1 B_{e x}$ & $0.1 B_{e x}$
\end{tabular}


simulation run, resulting in a standing wave. This set-up is similar to the situation in the ionospheric resonance region where the upgoing pump wave and the reflected wave coexist. Other than the pump waves, we assume a static density irregularity with a wave number $\pm k_{n}$. The parameters for the simulation are listed in Table 1. Here, the electron cyclotron frequency $\Omega_{e}$, and light velocity $c$, are bases in the simulation parameters. Other physical quantities such as intensity of external magnetic field, plasma temperature and density are decided by those parameters according to equations of physics. The periodic boundary conditions is assumed in both $x$ and $y$-directions of the simulation space.

The initial profiles of the wave fields in the simulation system is shown in Fig. 3. The direction of the external magnetic field is assumed to be parallel to the $y$-axis. The coherent field aligned irregularity can be seen in Fig. $3 \mathrm{a}$ which shows the electron density distribution. Ions are also displaced to form the identical distribution to electrons, so that the charge neutrality is established everywhere in the system. Figure $3 \mathrm{~b}$ and c shows two electric field components $\left(E_{x}, E_{y}\right)$ of the pump wave. The $E_{y}$ is the main longitudinal component of the wave. Note that the wave front is slightly oblique to the direction of the geomagnetic field.

Figure 4 shows the results of the simulation at time $\Omega_{e} t=1.12$, that is $\omega_{o} t \simeq 4.61$. As shown in Fig. 4a the electron density distribution stays almost stationary. The perpendicular component $E_{x}$ shown in Fig. $4 \mathrm{~b}$ is obviously given a small-scale modulation in the $x$ direction while $E_{y}$ shown in Fig. $4 \mathrm{c}$ and $E_{z}$ components are not affected. The identical modulation is also found in the perpendicular component of current density $J_{x}$. The wavelength of the modulation is close to that of the density irregularity. By comparing Fig. 3 with Fig. 4, we can find a great increase in the amplitude of $E_{x}$ component. This suggests that the density irregularity scattered the energy of the electromagnetic pump wave giving rise to the electrostatic wave.

Figure 5 shows $\boldsymbol{E} \cdot \boldsymbol{J}$ in the $2 \mathrm{D}$ wave number space $\left(k_{x}-k_{y}\right.$ space). A unit mode number $\left(k_{x}\right.$ or $\left.k_{y}=1\right)$ means the wavelength equals to the system length of the simulation space in the $x$ - or $y$-direction, respectively. The positive value of $\boldsymbol{E} \cdot \boldsymbol{J}$ (bright color) represents the wave damping, while the negative value (dark color) the wave growth. We find that energy conversion takes place from the pump wave $\left(k_{x}=4\right.$ and $\left.k_{y}=1\right)$ to the excited waves at $k_{x}=36$ and -28 , i.e., $k_{x}=4 \pm 32$ which correspond to $k_{o \perp} \pm k_{n}$ at the same $k_{y}(=1)$ as the pump wave. The matching condition in the $2 \mathrm{D}$ wave number space schematically shown in Fig. 6 is clearly satisfied. The energy of the excited mode is observed as $5 \%$ of the pump wave in Fig. 5.

The $E_{x}$ and $J_{x}$, according to Eq. (3), should oscillate with a frequency $\omega_{o} \pm \omega_{n} \simeq \omega_{o}$. The frequency found in the simulation is shown in the $\omega-k_{x}$ diagrams in Fig. 7 for $E_{x}$ and $E_{y}$. In Fig. 7a, we can see the pump wave $\left(\omega / \Omega_{e} \simeq 4.8, k_{x}=k_{o \perp}=4\right), \quad$ a $\mathrm{Z}$-X mode wave $\left(\omega / \Omega_{e} \simeq 3.8, k_{x}=k_{o \perp}\right)$, and the excited electrostatic (ES) waves. The Z-X mode wave is a left elliptically polarized, obliquely propagating wave as a L-O mode wave while the frequency is lower than plasma frequency. The frequency of the excited ES waves is almost the same as $\omega_{o}$ and their mode numbers are $k_{x}=k_{o \perp} \pm k_{n}$ $(=36,-28)$. These ES waves should be the UH waves excited by the direct conversion (DC). The frequency and mode numbers of the excited UH waves satisfy the matching condition (Eq. 4) as suggested by the theory.

The other weak ES waves are also seen at $\omega / \Omega_{e} \simeq 3.5, k_{x}=k_{o, \perp} \pm k_{n}$. This is due to a Z-X mode resulting from some deviation of the initial condition of the pump L-O mode wave from the exact polarization given by the analytic linear dispersion relation at $\left(\omega, k_{x}\right)=\left(3.8 \Omega_{e}, 4\right)$. It is technically difficult to initialize the thermal particles containing the sloshing motion of the L-O mode wave only. Actually in Fig. 7b, the Z-X electromagnetic component is seen in the simulation result. The frequency of the weak ES waves which is on one branch of the Bernstein modes is shifted from the Z$\mathrm{X}$ mode wave by $\Delta \omega=0.3 \Omega_{e}$ which is very close to the ion plasma frequency. This strongly suggests that the three wave process among the Z-X, the weak ES wave and an ion acoustic wave took place in the system. The matching condition for the frequency and mode number is shown as $3.8 \Omega_{e}=3.5 \Omega_{e}+0.3 \Omega_{e}$, and $k_{o \perp}=$ $\left(k_{o \perp}-k_{n}\right)+k_{n}$.

The situation in which two different frequency pump waves are simultaneously transmitted is not necessarily the same as that of ionospheric heating experiments. The results, neverthless, reproduce the physical processes of the electrostatic wave excitation related to the existence of small-scale density irregularity. The DC process arises from the L-O mode pump, the other three wave process is separately due to the Z-X mode pump. Since the L-O mode pump wave and Z-X mode wave are at the same wave number, there exists no resonance condition involving the density irregularity with finite wave numbers. Therefore, we assume there is no direct coupling between the pump wave and the Z-X mode wave.

To study the dependence of the excited wave amplitude on various parameters, we performed 1-dimensional simulation runs to reduce the CPU time. In the code, physical quantities such as particle velocity and electric field have 3-dimensional components same as the 2D code. Therefore the code can treat elliptically polarized pump waves. There are some minor differences between 2D and 1D simulation models. In the 1D simulations $\boldsymbol{k}_{n}$ is regarded as parallel to $\boldsymbol{k}_{\boldsymbol{o}}$, so that irregularity is slightly oblique to $\boldsymbol{B}_{e x}$. However, the excitation process observed in the 1D model is essentially the same as in the $2 \mathrm{D}$ simulation. The parameters for the simulations are also listed in Table 1.

We evaluate the amplitude of the excited wave normalized to that of the pump wave $|E| /\left|E_{o}\right|$. The amplitude of the excited wave $|E|$ is the maximum value of $E_{x}$ with the mode $k_{o}+k_{n}$. It is about 20 times as large as the thermal noise level in a simulation run without the pump wave. Here, resolutions of the frequency and the mode number are $0.3 \Omega_{e}$, and $0.125 k_{o}$, respectively. Maximum values should be $61.2 \Omega_{e}$ and $512 k_{o}$ which correspond to greater than $16 k_{n}$, in the simulation. 


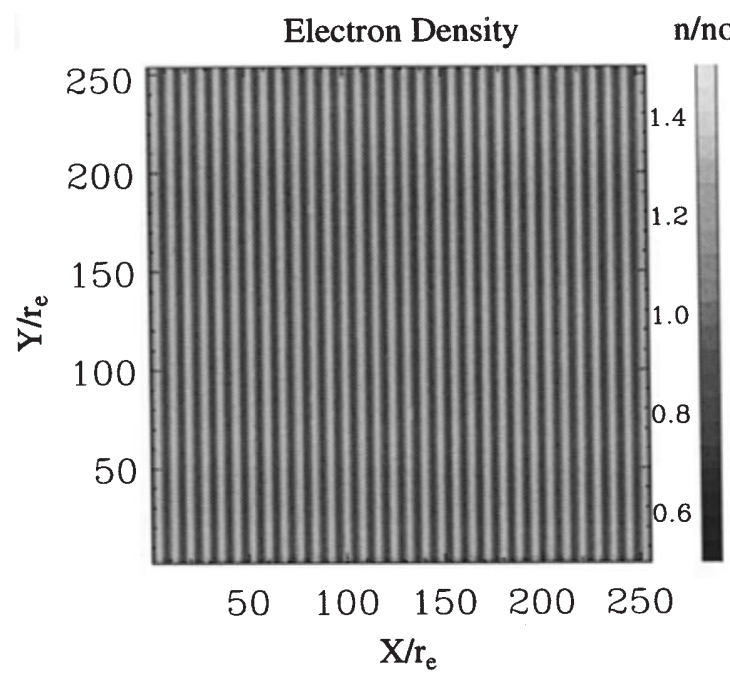

a

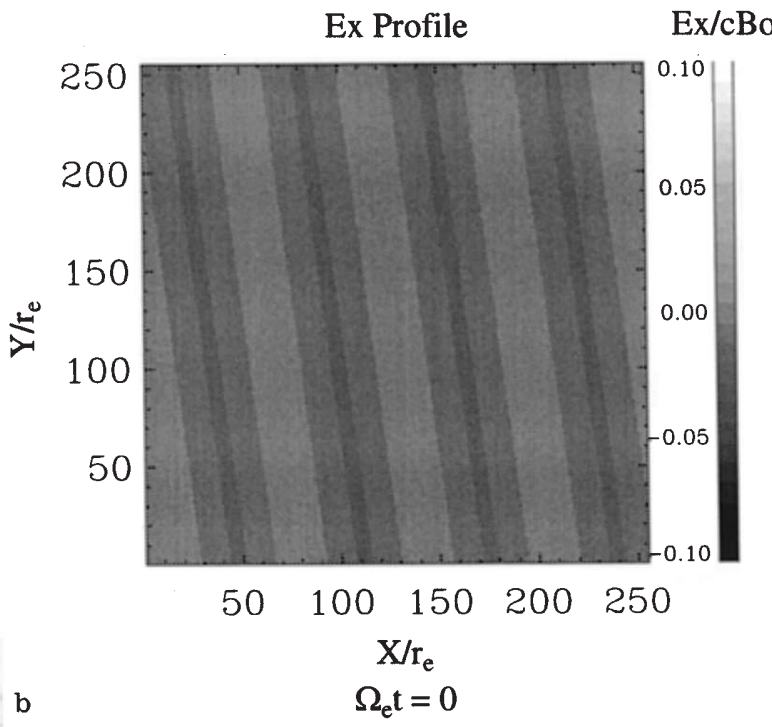

Ey Profile

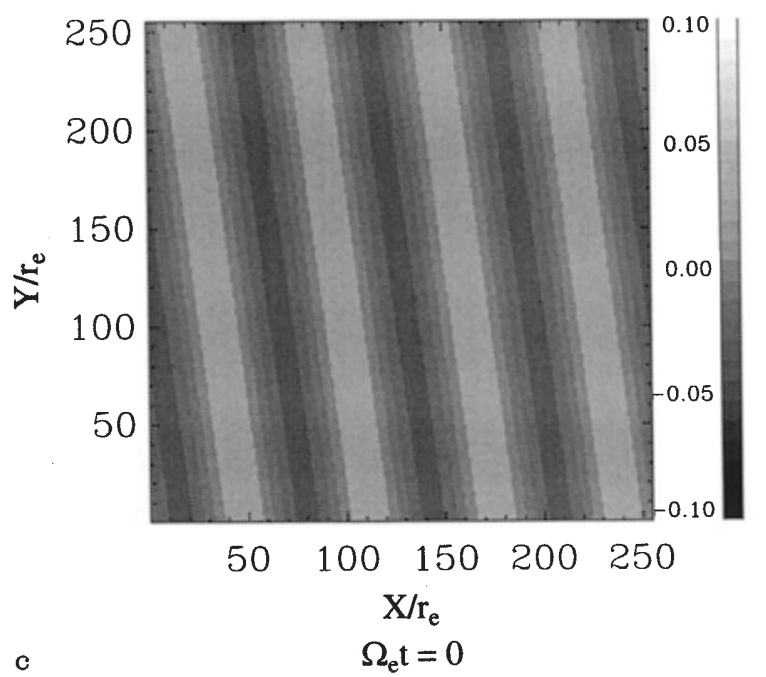

Fig. 3a-c. Contour plots of a electron density $n_{e}$, b perpendicular electric field $E_{x}$, and $\mathbf{c}$ parallel electric field $E_{y}$ in the $x-y$ simulation plain at $t=0.0$
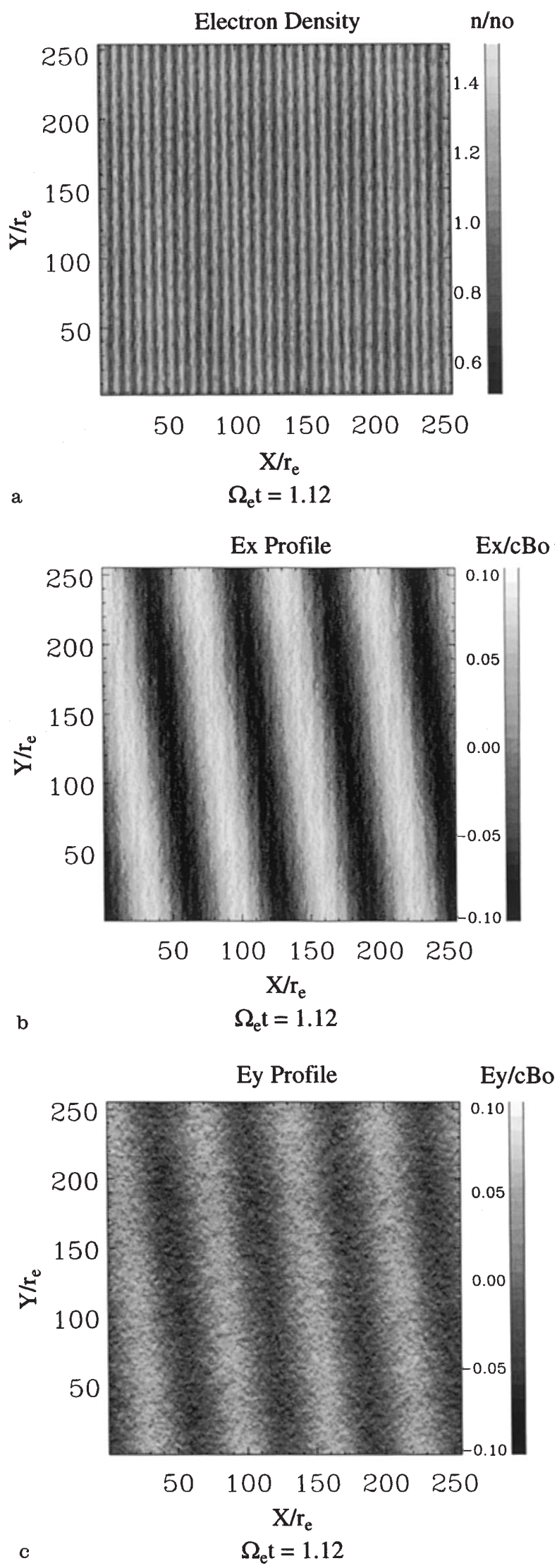

Fig. $4 \mathbf{a}-\mathbf{c}$. Contour plots of $\mathbf{a} n_{e}, \mathbf{b} E_{x}, \mathbf{c} E_{y}$, at $t=1.12 / \Omega_{e}$. A strong coupling with the density irregularity at the short wavelength is found in $E_{x}$ 


\section{$\mathrm{E} \cdot \mathrm{J}$}

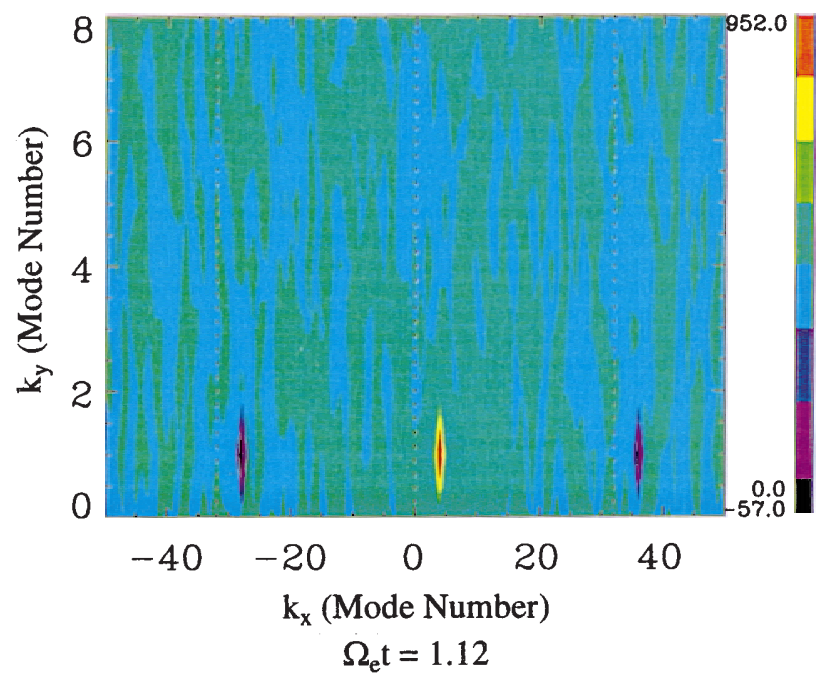

Fig. 5. $\boldsymbol{E} \cdot \boldsymbol{J}$ in $2 \mathrm{D}$ wave number space at $t=1.12 / \Omega_{e}$. The positive $\boldsymbol{E} \cdot \boldsymbol{J}$ (bright color) represents the wave damping, while the negative values (dark color) correspond to the wave growth. The energy conversion from the pump wave $\left(k_{x}=4, k_{y}=1\right)$ to the excited waves $\left(k_{x}=36,-28, k_{y}=1\right)$ occurs under the matching condition in the wave number space

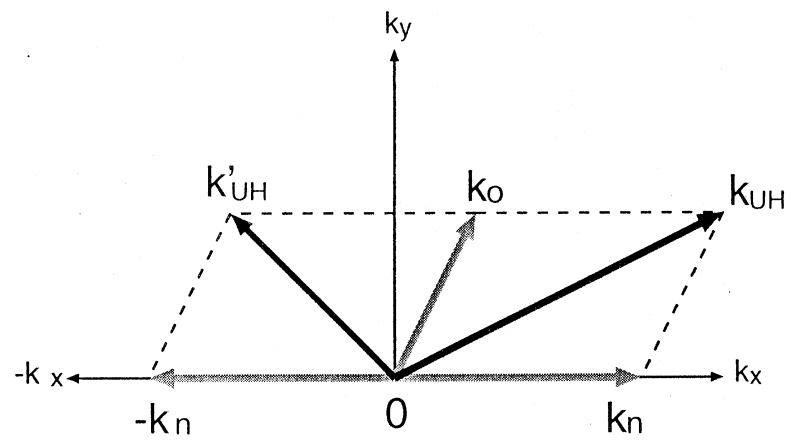

Fig. 6. Schematic illustration of the matching condition in the $2 \mathrm{D}$ wave number space. The wave number of the excited wave $\boldsymbol{k}_{\boldsymbol{U}}$ is determined by the wave number of the pump wave $\boldsymbol{k}_{\boldsymbol{o}}$ and that of the density irregularity $\boldsymbol{k}_{\boldsymbol{n}}$

Numerical diffusion can be neglected because both of $\omega / \omega_{\max }$ and $k / k_{\max } \ll 1$. The amplitude of the pump wave $\left|E_{o}\right|$ is evaluated by $\sqrt{E_{x}^{2}+E_{y}^{2}+E_{z}^{2}}$ for the mode $k_{o}$ at the beginnings of the simulation run. Since the $E_{o}$ is a sum of the pump L-O and Z-X modes, the actual ratios of the excited waves to the L-O mode pump wave are $1 \sim 6 \%$ larger than the values in the following results. Figure 8 shows the relative amplitude as functions of the parameters $\left(\lambda, \theta\right.$ and $\left.n_{1} / n_{o}\right)$ mentioned in Sect. 2 . In Fig. 8 a we changed $\lambda$ defined as $k_{\perp}^{2} r_{e}^{2}$, varying $k_{n}$ which corresponds to $k_{\perp}$ in the range from $2 k_{o}$ to $32 k_{o}$ which corresponds to the range of $2 \pi / k_{n}$ from $64 r_{e}$ to $4 r_{e}$. The thermal velocity is fixed to $0.053 \mathrm{c}$ except for one case expressioned by a symbol ' + ' in Fig. 8a, in which the thermal velocity is $0.02 \mathrm{c}$. A dashed line in Fig. $8 \mathrm{c}$ is a linearly fitted line of the data observed in the simulations. The maximum relative amplitude was found to be $37 \%$ (13\% for energy) for a set of parameters of $\theta=70$,
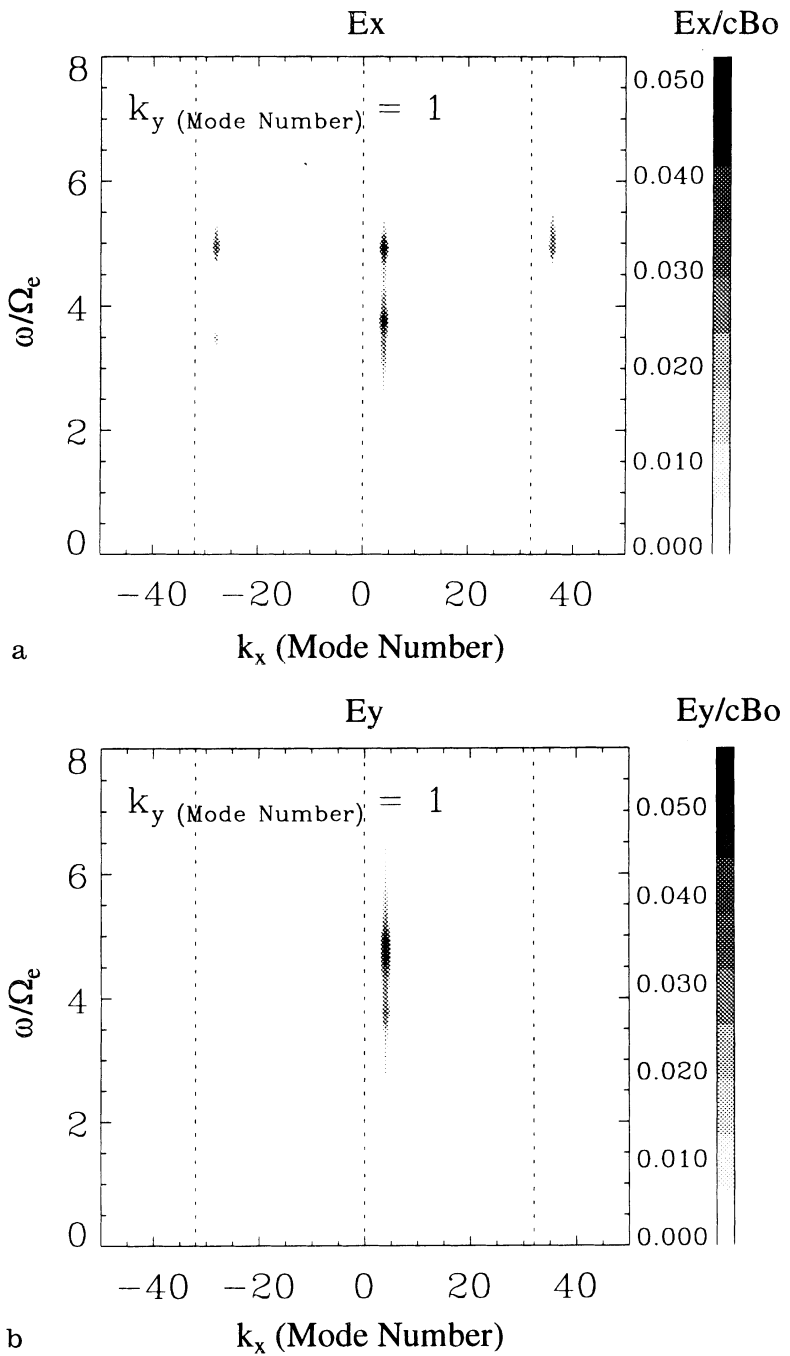

Fig. 7a,b. $\omega-k_{x}$ diagrams of a perpendicular electric field $E_{x}, \mathbf{b}$ parallel electric field $E_{y}$. The pump electromagnetic wave $\left(\omega=\omega_{o} \simeq 4.8 \Omega_{e}, k_{x}=k_{o \perp}=4\right), \quad$ the $\mathrm{Z}-\mathrm{X}$ mode wave $\left(\omega \simeq 3.8 \Omega_{e}, k_{x}=k_{o \perp}\right)$, and the excited electrostatic waves $\left(\omega=\omega_{o}, k_{x}=k_{o \perp} \pm k_{n}=36,-28\right)$ are found in the perpendicular electric field, while the parallel one shows the pump wave and the Z-X mode wave

$\lambda=0.037, \tilde{n}_{1} / n_{o}=0.2$ (see Fig. $8 \mathrm{~b}$ ). Significant intensity dependence was not found between the range of $\boldsymbol{B}_{o} / \boldsymbol{B}_{e x}=0.01 \sim 0.2$, where $\boldsymbol{B}_{o} / \boldsymbol{B}_{e x}$ is a ratio of the pump magnetic amplitude to the external magnetic field. Figure 9 shows, on the other hand, the results of numerical calculations of Eq. (7) as functions of $\lambda$ and $\theta$, where $|D|$ is evaluated by the linear theory. The calculations were made for the parameter set shown in the figure which is the same as in the simulation. Figure 9a shows essentially identical characteristics as Fig. 2 of the paper by Antani et al. (1991) in which the parameters correspond to the real ionospheric conditions. We find that the values of $|E| /\left|E_{o}\right|$ in Fig. 9 are much larger than those of Fig. 8. The very efficient conversion $\left(|E| /\left|E_{o}\right|>1\right)$ as predicted by the theory is not observed in the simulations. For parameter dependences on $\lambda$, shown in Fig. 8a, the relative amplitude decreases as $\lambda$ increases from 0.02 to 0.6 . The range of $\lambda$ in the 

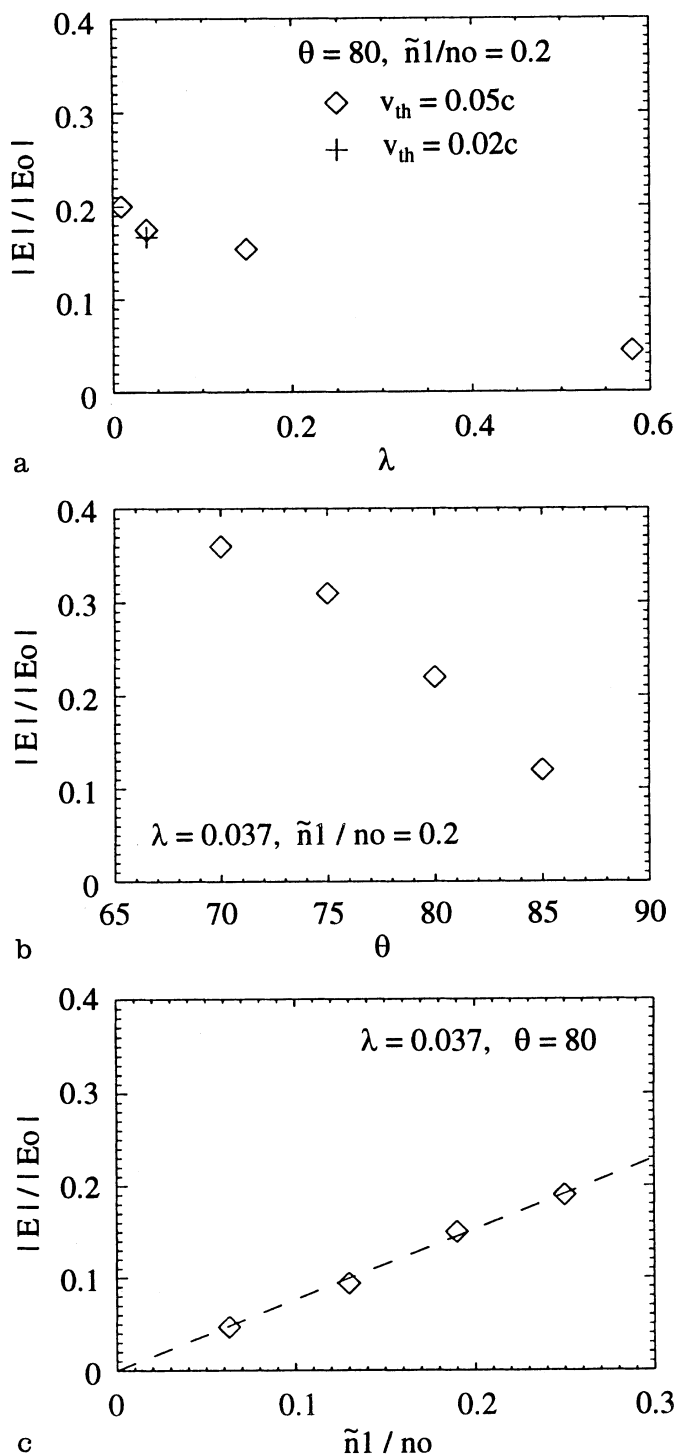

Fig. 8a-c. Ratios of the excited wave amplitude to the pump amplitude given by the simulations. The variable parameters are $\mathbf{a} \lambda$ and $v_{t h}, \mathbf{b} \theta$, and $\mathbf{c} n_{1}$, respectively. The dashed line in $\mathbf{c}$ is a linearly fitted line

simulation is located on the right side of the peak in Fig. 9a. It agrees qualitatively with the linear analysis. Because of the damping rate of the $\mathrm{UH}$ wave within this range, the amplitude $|E|$ decreases as $\lambda$ closes to 1 , the wavelength closes to the Larmor radius. For dependence on $\tilde{n}_{1} / n_{o}$ shown in Fig. 8c, the observed data is almost in agreement with the linearly fitted line as shown in the predictions by the theory. As for the dependence on $\theta$, the curvatures in Figs. $8 \mathrm{~b}$ and $9 \mathrm{~b}$ seem to be opposite. This suggests nonlinear effects on $|D|$ in the simulation.

The difference in the curvature and amplitude between the simulations and the linear theory is due to the nonlinear dispersion effect neglected in the theory. The evaluated value of $|D|$ using the coefficient of the fitted line in Fig. 8c is 0.21 , while by the linear theory it is 0.012 . The ratio of $|E| /\left|E_{o}\right|$ becomes exponentially
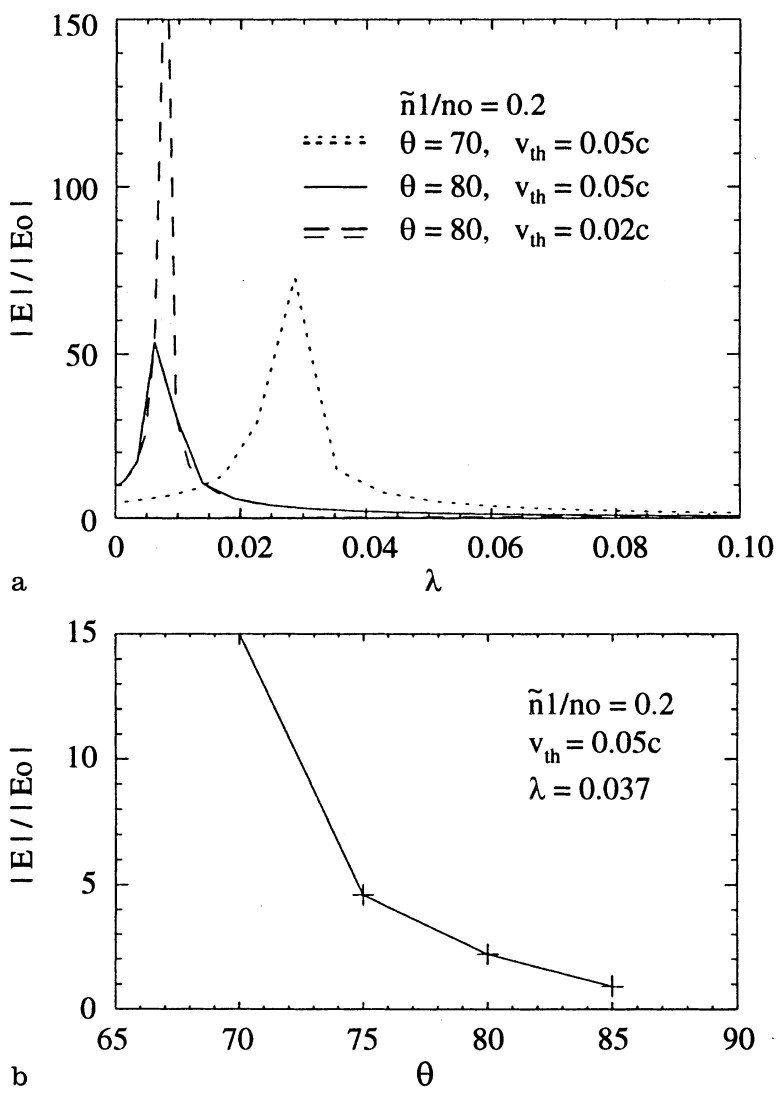

Fig. 9a,b. Calculation of the ratio of the UH wave amplitude to the pump wave amplitude as functions of $\mathbf{a} \lambda, \mathbf{b} \theta$

large when the value of $|D|$ close to zero in the linear theory shown Fig. 9b. The simulation results in Fig. 8b, however, suggest that a finite nonlinear term of $|D|$ is operating to make the term of $\cos \theta$ in Eq. (7) more influential in the $\theta$ dependence. In the simulation, as well as the real ionospheric system, we cannot neglect the nonlinear effects induced by the large amplitude pump waves. There has been no direct measurement of the conversion efficiency in the ionosphere. The results of the present simulation should give an important clue for estimation of the efficiency of the DC process, but its validity as a generation mechanism of SEE has to be tested in a future ionospheric heating experiment accompanied by an in-situ measurement of the electrostatic waves in the ionosphere.

\section{Discussions and conclusions}

We performed particle-in-cell simulations to investigate the possibility of the direct conversion process in the ionospheric heating experiments where the pump wave stimulates the SEE (stimulated electromagnetic emission observed during the heating) via the excitation of the ES waves. The simulation results reproduced the features of the DC process. The UH waves are excited from the L-O mode pump wave in the plasma where a coherent density irregularity exists. We confirmed that the scale length of the irregularity exactly determine the wave 
number of the excited wave. If there exists multi-scale or broad band irregularity, the wave number of the excited wave must be selected according to Eq. (7).

A few $10 \%$ of the pump energy is readily converted into the $\mathrm{UH}$ wave in the $\mathrm{UH}$ resonance region where various density irregularities exist naturally or artificially. The maximum amplitude of the excited wave was recorded as $37 \%$ of the pump amplitude, under an optimum condition, that was found from the several simulation runs where we varied propagation angle of the pump wave, scale length of the density irregularity, degree of the irregularity, and thermal velocity of the plasma. The qualitative nature of the conversion fundamentally depends on the condition in the interaction region as predicted by theoretical work (Antani et al., 1991). The simulation resutls reveal, however, that the conversion efficiency $\left(|E| /\left|E_{o}\right|\right)$ is much less than the prediction due to nonlinear effects. Thermal velocity was found to be almost kept constant throughout the simulations. This excludes a possibility of the change of the dispersion nature due to plasma heating, leaving another possibility that the high-power pump does change the critical resonance condition, where $|D|$ is closes to zero, according to the linear theory.

We have tried two other cases with different pump frequency. The pump frequency in these cases was chosen to be the integer multiple of the cyclotron frequencies. This choice was based on the experimental fact that the SEE is observed when the pump frequency is close to the integer multiple of the electron cyclotron frequency. The result of our simulations, however, did not show any excitation of the electrostatic waves. A possible reason for this is the difficulty of satisfying the frequency and wave number matching condition involving the higher branch of the electron Bernstein modes above the UH frequency. This is simply because the dispersion relation for those branches is allowed only in the very narrow frequency band between the integer multiple of the electron cyclotron frequency and the maximum allowed frequency of the mode.

The present study, however, demonstrated via simulation that the DC process can actually happen even if we include the relevant nonlinear effects (such as the frequency shift and mode-couplings). The DC process produces the ES wave which makes it possible to generate the SEE through the second step nonlinear wave-wave coupling.
Acknowledgements. The computer simulations in this work were performed on the KDK computer system at Radio Atmospheric Science Center, Kyoto University.

Topical Editor D. Alcaydé thanks S. C. Chapman and another referee for their help in evaluating this paper.

\section{References}

Antani, S. N., N. N. Rao, and D. J. Kaup, Direct conversion of ordinary mode into upper-hybrid wave by density irregularities in the ionosphere, Geophys. Res. Lett., 18, 2285-2288, 1991.

Antani, S. N., D. J. Kaup, and N. N. Rao, Excitation of upper hybrid waves from ordinary-mode electromagnetic waves via density gradient in the ionosphere, J. Geophys. Res., 101, A12, 27 035-27 041, 1996.

Goodman, S., B. Thidé, and L. Erukhimov, A combined parametric and conversion mechanism for upshifted stimulated electromagnetic emissions, Geophys. Res. Lett., 20 (8) 735-738, 1993.

Hedberg A, H. Derblom, B. Thidé, H. Kopka, and P. Stubbe, Observations of HF backscatter associated with the heating experiment at Troms, RadioSci., 18, 840-850, 1983.

Kagan, L. M., and V. L. Frolov, Significance of field-aligned currents for F-region perturbations, J. Atmos. Terr. Phys., 58, (13), 1465-1474, 1996.

Leyser, T. B., B. Thidé, H. Derblom, A. Hedberg, B. Lundborg, P. Stubbe, and H. Kopka., Stimulated electromagnetic emission near cyclotron harmonics in the ionosphere. Phys. Rev. Lett., 63, 1145-1147, 1989.

Leyser, T. B., B. Thidé, H. Derblom, A. Hedberg, B. Lundborg, P. Stubbe, and H. Kopka, Dependence of stimulated electromagnetic emission on the ionosphere and pump wave, $J$. Geophys. Res., 95, 17 233-17 244, 1990.

Leyser, T. B., B. Thidé, M. Waldenvik, and S. Googman, Spectral structure of stimulated electromagnetic emissions between electron cyclotron harmonics, J. Geophys. Res., 98, 17 597-17 606, 1993.

Leyser, T. B., B. Thidé, M. Waldenvik, E. Veszelei, V. L. Frolov, S. M. Grach, and G. P. Komrakov, Dawnshifted maximum features in stimulated electromagnetic emission spectra, J. Geophys. Res., 99, 19 555-19 568, 1994.

Morales, G. J., S. N. Antani, and B. D. Fried, Direct conversion of a fast wave into ion Bernstein modes caused by density fluctuations. Phys. Fluids., 28, (11), 3302-3312, 1985.

Matsumoto, H., and Y. Omura, Particle simulation of electromagnetic waves and its application to space plasmas, in Computer simulations of space plasmas, ed. H. Matsumoto, T. Sato, Terra Publications, 1984.

Thidé, B., A. Hedberg, J. A. Fejer, and M. P. Sulzer, First observations of stimulated electromagnetic emission at Arecibo, Phys. Rev. Lett., 16, 369-372, 1989.

Wong, A. Y., G. J. Morales, D. Eggleston, J. Santoru, and R. Behnke, Rapid conversion of electromagnetic waves to electrostatic waves in the ionosphere, Phys. Rev. Lett., 47, 1340-1343, 1981. 